\title{
DÁBITOS LECTORES Y COMPETENCIAS BÁSICAS EN EL ALUMNADO DE EDUCACIÓN SECUNDARIA OBLIGATORIA ${ }^{1}$
}

\author{
(READING HABITS AND BASIC COMPETENCES IN STUDENTS OF \\ COMPULSORY SECONDARY EDUCATION)
}

Javier Gil Flores

Universidad de Sevilla

\section{RESUMEN}

El artículo se centra en el análisis de la relación entre hábitos lectores y aprendizaje. Para ello hemos recurrido a la metodología de investigación mediante encuesta y se han tomado en consideración resultados de las pruebas de diagnóstico, aplicadas en los centros educativos andaluces a principios del curso escolar 2008/2009. Han participado en el estudio un total de 82.961 alumnos y alumnas de centros educativos públicos y concertados, matriculados en tercer curso de la ESO. Los resultados muestran que una lectura frecuente de textos literarios, con un propósito recreativo, se asocia a niveles altos de competencia en los ámbitos matemático, lingüístico y científico. En cambio, la frecuencia con que se practica una lectura instrumental, dirigida a la mera localización de información o a la participación en situaciones de comunicación a través de Internet, no se vincula a diferentes niveles de aprendizaje.

\section{ABSTRACT}

This paper focuses on the analysis of the relationship between reading habits and learning. We have used the survey as main research methodology, taking into account the results of the diagnostic tests applied on Andalusian schools at the beginning of the academic year 2008/2009. The subjects in the sample are 82,921 students of public and private stataly financed schools pertaining to the third year of Compulsory Secondary Education. The study re- 
veals that a frequent reading of literary texts with a recreational purpose is associated to high levels of competence in mathematical, linguistic and scientific domains. However, the frequency of instrumental reading, just devoted to the finding of information or the participation in communicative situations in Internet, is not related to different learning levels.

\section{INTRODUCCIÓN}

La importancia de la lectura como herramienta para el acceso a la información sitúa a la competencia lectora entre los aprendizajes básicos que se esperan en los estudiantes al término de la escolarización obligatoria. Probablemente la lectura constituye la práctica cultural que goza de un mayor reconocimiento, debido a su papel como instrumento para el desarrollo de una mentalidad crítica y para la socialización de los ciudadanos (Fernández, 2005). La competencia lectora es un atributo indispensable para que los ciudadanos puedan desenvolverse en el medio social, y constituye una pieza clave para el enriquecimiento intelectual, la adquisición de aprendizajes y el acceso a la cultura.

Pero además, los lectores habituales mantienen actitudes positivas hacia la lectura y leen con el fin de obtener un disfrute personal (Mullis, Kennedy, Martin y Sainsbury, 2006), encontrando así una interesante y enriquecedora actividad a realizar en su tiempo de ocio. Al considerar aquí los hábitos lectores haremos referencia a un tipo de lectura que los individuos realizan al margen de sus actividades laborales o académicas, por iniciativa propia y con plena libertad para la elección de los materiales de lectura.

A pesar del valor atribuido al hábito lector, la presencia de éste entre los ciudadanos de nuestro país ha retrocedido ligeramente en los últimos años, rompiendo la tendencia ascendente registrada durante casi una década. Así se deduce del Barómetro de Hábitos de Lectura y Compra de Libros, que desde 2001 elabora trimestralmente la Federación de Gremios de Editores de España con el patrocinio de la Dirección General del Libro, Archivos y Bibliotecas del Ministerio de Cultura. De acuerdo con los datos provenientes de este estudio, el porcentaje de españoles mayores de 14 años que suelen leer libros en su tiempo libre con una periodicidad diaria o semanal era un 56,9\% en 2007 y ha descendido en 2009 hasta el 54,3\% (FGGE, 2009). Quienes se declaran no lectores (no leen nunca o casi nunca) representaban en 2007 el 43,1\% de la población y el 45,7\% en 2009. Con estas cifras, el índice de lectura en nuestro país sigue estando lejos del registrado en la Unión Europea, donde en 2007 un 71\% de la población afirmaba haber leído al menos un libro en el último año (European Comission, 2007). 
En el ámbito escolar, la lectura frecuente se encuentra indiscutiblemente asociada al grado de desarrollo que los escolares alcanzan en competencia lectora. El compromiso de los estudiantes con la lectura y el interés por la misma se han revelado como dos de los factores que en mayor medida contribuyen a explicar el rendimiento en comprensión lectora (Linnakylä y Välijärvi, 2006). Centrándonos en habilidades lectores específicas, se ha demostrado la existencia de una relación significativa entre la cantidad de tiempo dedicada a la lectura fuera de la escuela por el alumnado de educación primaria y las puntuaciones logradas en deletreo, vocabulario, reconocimiento de palabras, fluidez verbal (Cunnigham y Stanovich, 1991) o comprensión lectora (Anderson, Wilson y Fielding, 1988). Estudios recientes han venido a confirmar que los hábitos lectores predicen las habilidades lectoras del alumnado, tanto en los primeros años de la educación primaria (Leppanen, Aunola, y Nurmi, 2005), como entre adolescentes (Conlon y otros, 2006).

Teniendo en cuenta los relativamente bajos hábitos lectores que se registran entre los jóvenes españoles, no deben extrañar los poco satisfactorios resultados obtenidos al evaluar la competencia lectora de nuestros escolares. Así lo han puesto de manifiesto estudios internacionales en los que ha participado nuestro país desde los años 90. Como señala Martín (2008), en las evaluaciones PISA (Programme Internactional form Student Assessment) y PIRLS (Progress in International Reading Literacy Study), los resultados logrados por los estudiantes españoles en comprensión lectora vienen siendo inferiores a los obtenidos por la mayoría de los países europeos participantes en la evaluación, mostrando un panorama que ha de valorarse negativamente. Además, la periodicidad de estas evaluaciones permite apreciar la evolución que se registra con el paso del tiempo. El análisis de los resultados obtenidos en las sucesivas evaluaciones PISA realizadas en 2000, 2003 y 2006 muestra en esta última edición un descenso generalizado de la competencia lectora en todos los países, y particularmente en España, donde la puntuación promedio descendió a 461 puntos, bastante por debajo de la media de los países de la OCDE, situada en 492 .

Pero los hábitos de lectura no sólo están asociados con la competencia lectora, sino en general con los aprendizajes escolares. Los hábitos de lectura no constituyen únicamente un elemento favorecedor de la técnica lectora; además contribuyen a través de ésta al logro de aprendizajes en diferentes ámbitos. El dominio de la técnica lectora se considera un prerrequisito básico para el éxito en la mayor parte de las disciplinas escolares. De ahí que numerosos estudios hayan demostrado la relación entre la competencia lectora y el rendimiento (Cromley, 2009; Meneghetti, Carretti y De Beni, 2006; Nolen, 2003; O’Reilly y McNamara, 2007; Savolainen y otros, 2008; 
Topping, Samuels y Paul, 2007). También en nuestro país la asociación entre lectura y aprendizaje ha sido confirmada en investigaciones llevadas a cabo sobre este tema, en las que se han encontrando correlaciones significativas entre las calificaciones escolares y variables, tales como el gusto por la lectura (Molina, 2006) o la comprensión lectora (González, 1996; Fiz y otros, 2000b).

\section{PLANTEAMIENTO DEL PROBLEMA}

Teniendo en cuenta las reflexiones anteriores, en el presente trabajo nos hemos planteado el estudio de la incidencia que poseen los hábitos lectores sobre el dominio de las competencias básicas en los ámbitos matemático, lingüístico y científico. Concretamente, hemos analizado las diferencias existentes en el nivel competencial del alumnado de Educación Secundaria Obligatoria en función de la frecuencia con que realizan diferentes tipos de lecturas, excluyendo las que se realizan sobre materiales curriculares empleados en el contexto de los procesos de enseñanza-aprendizaje.

Nuestro interés está, por tanto, en determinar la relación existente entre los hábitos de lectura, como modo de ocupar el tiempo libre extraescolar, y el desarrollo alcanzado en diferentes competencias básicas. Más allá de la competencia lectora, los hábitos lectores podrían ser favorecedores de otros aprendizajes escolares, incluyendo entre éstos la competencia del sujeto en comunicación lingüística, la competencia matemática o la competencia científica. Además, pretendemos determinar qué tipos de lecturas realizadas por los estudiantes se asocian al logro de un mayor nivel en las competencias básicas consideradas, entendiendo que no todas las lecturas responden al mismo propósito ni generan en los lectores procesos cognitivos de la misma naturaleza, por lo que unas podrían contribuir en mayor medida que otras a la adquisición de aprendizajes.

\section{METODOLOGÍA}

Los datos correspondientes a las diferentes variables consideradas en el presente estudio han sido recogidos en el marco de la evaluación de diagnóstico llevada a cabo en Andalucía en el curso 2008/2009, evaluación en la que hemos participado en virtud de un convenio de colaboración firmado entre la Consejería de Educación y la Universidad de Sevilla. Con esta evaluación de diagnóstico se responde a lo establecido en la Ley Orgánica 2/2006 de 3 de mayo, de Educación, por la que todos los centros han de realizar una evaluación de diagnóstico de las competencias básicas alcanza- 
das por el alumnado. De cara a tener en cuenta los factores contextuales que pudieran incidir en el aprendizaje escolar, además de evaluar el desarrollo de competencias por parte del alumnado se ha recogido información sobre un conjunto amplio de variables, utilizando para ello cuestionarios que fueron respondidos tras la realización de las pruebas. Entre estas variables se encuentran los hábitos lectores de los estudiantes objeto de evaluación.

El estudio se ha llevado a cabo, por tanto, adoptando un diseño de investigación ex-post facto, de carácter descriptivo, basado en la medición del nivel logrado por los estudiantes en competencias básicas y en la aplicación de métodos de encuesta. La población considerada la constituye el alumnado que había concluido el curso segundo de Educación Secundaria Obligatoria, encontrándose en el mes de octubre de 2008 matriculado en tercer curso de este nivel educativo, en centros educativos públicos y concertados de la Comunidad Autónoma de Andalucía. La población estudiada asciende a un total de 82.961 alumnos y alumnas, cuya distribución por provincias y titularidad de los centros a los que asisten se muestra en la tabla 1.

\begin{tabular}{|l|c|c|c|}
\hline & \multicolumn{2}{|c|}{ TITULARIDAD } & \\
\hline Provincia & $\begin{array}{c}\text { Centros } \\
\text { públicos }\end{array}$ & $\begin{array}{c}\text { Centros } \\
\text { privados }\end{array}$ & $\begin{array}{c}\text { Total de } \\
\text { estudiantes }\end{array}$ \\
\hline Almería & 5582 & 802 & 6384 \\
Cádiz & 9466 & 3179 & 12645 \\
Córdoba & 6395 & 2014 & 8409 \\
Granada & 6768 & 2690 & 9458 \\
Huelva & 4245 & 962 & 5207 \\
Jaén & 6201 & 1660 & 7861 \\
Málaga & 10461 & 3670 & 14131 \\
Sevilla & 14059 & 4807 & 18866 \\
\hline Todas las & 63177 & 19784 & 82961 \\
provincias & & & \\
\hline
\end{tabular}

Tabla 1. Distribución de la población de estudiantes evaluados por provincias y titularidad de los centros. 
Los instrumentos utilizados en la recogida de datos acerca del nivel de competencia de los estudiantes han sido tres pruebas de evaluación, administradas a la totalidad de los alumnos y alumnas de la población estudiada. Dichas pruebas fueron aplicadas en el mes de octubre de 2008, dedicando para ello en cada centro tres sesiones de hora y media, que tuvieron lugar en tres días consecutivos. Las preguntas planteadas se basaban en la presentación previa de casos o situaciones similares a las que el alumno puede encontrar en su vida cotidiana, y cuya resolución requería poner en juego competencias adquiridas en el ámbito matemático (competencia matemática), lingüístico (competencia en comunicación lingüística) y científico (competencia en el conocimiento e interacción con el mundo físico y natural). De acuerdo con la regulación hecha por la Administración educativa andaluza sobre el procedimiento de evaluación, el profesorado de los centros ha sido el encargado de aplicar y corregir las pruebas. Las pruebas de evaluación generaron puntuaciones directas, que posteriormente fueron transformadas a una escala de media $500 \mathrm{y}$ desviación típica 100.

La información sobre hábitos lectores se recogió mediante una pregunta incluida en los cuestionarios de contexto administrados a los alumnos y alumnas participantes en la evaluación. Esta pregunta pedía a los encuestados que indicaran con qué frecuencia realizaban siete diferentes tipos de lecturas fuera del colegio: "leo tebeos", "leo cuentos o novelas cortas", "leo libros (colecciones juveniles, sobre deportistas, viajes...)", "leo revistas", "leo periódicos", "leo juegos de preguntas y respuestas", "leo en Internet". La escala de respuestas contemplaba cuatro opciones ordenadas, desde la que implica menor frecuencia de lectura hasta la que corresponde a quienes leen a diario: "nunca o casi nunca", "una o dos veces al mes", "una o dos veces a la semana", "todos o casi todos los días".

Tras llevar a cabo el proceso de encuesta, se recogieron un total de 72.570 cuestionarios. Las respuestas obtenidas para los cuestionarios de contexto fueron codificadas y tabuladas para su análisis. Los valores numéricos resultantes se incluyeron, junto con las puntuaciones obtenidas en las pruebas de evaluación de diagnóstico, en una matriz numérica única.

El análisis de los datos se ha basado en la aplicación de diversos tipos de técnicas estadísticas. Se ha partido de una descripción de los hábitos lectores del alumnado, recurriendo a distribuciones de frecuencias. Mediante análisis factorial, se han reducido a dos modalidades los siete tipos de lecturas sobre las que han informado los alumnos. Tomando las puntuaciones en los dos factores resultantes, se ha dividido a los sujetos en cuatro grupos que se corresponden con cuatro niveles de frecuencia en cada una de las modalidades de lectura. Para cada grupo se han calculado los niveles medios lo- 
grados por los alumnos en las tres competencias consideradas, y se han representado éstos mediante diagramas de barras.

\section{RESULTADOS}

\subsection{Hábitos lectores del alumnado}

Las respuestas de los estudiantes andaluces de tercero de la ESO revelan que los hábitos lectores, en general, no se encuentren muy extendidos entre ese colectivo. De acuerdo con los resultados mostrados en la tabla 2, la lectura de literatura es una práctica ajena a una buena parte del alumnado. Así, aproximadamente la mitad de los encuestados $(51,30 \%)$ no lee cuentos o novelas cortas nunca o casi nunca, y un $28,68 \%$ no lee libros. La lectura de tebeos está muy poco extendida, con un 79,55\% de los estudiantes que se declara no lector de este tipo de textos.

\begin{tabular}{|l|c|c|c|c|}
\hline $\begin{array}{l}\text { Tipo de } \\
\text { lecturas }\end{array}$ & $\begin{array}{c}\text { Nunca o casi } \\
\text { nunca }\end{array}$ & $\begin{array}{c}1-2 \text { veces } \\
\text { al mes }\end{array}$ & $\begin{array}{c}1-2 \text { veces } \\
\text { a la semana }\end{array}$ & $\begin{array}{c}\text { Todos o casi } \\
\text { todos los días }\end{array}$ \\
\hline Tebeos & 79,55 & 12,43 & 6,03 & 1,99 \\
\hline $\begin{array}{l}\text { Cuentos o } \\
\text { Novelas cortas }\end{array}$ & 51,30 & 30,61 & 12,88 & 5,21 \\
\hline $\begin{array}{l}\text { Libros (juveni- } \\
\text { les, deportes, } \\
\text { viajes...) }\end{array}$ & 28,68 & 31,52 & 25,55 & 14,25 \\
\hline $\begin{array}{l}\text { Revistas } \\
\text { Juegos de pre- } \\
\text { guntas y } \\
\text { respuestas }\end{array}$ & 38,45 & 32,18 & 34,22 & 12,10 \\
\hline Internet & 13,72 & 12,49 & 25,35 & 5,91 \\
\hline
\end{tabular}

Tabla 2. Frecuencia con que se realizan diferentes tipos de lecturas (porcentajes)

Contrasta con estos resultados la situación en lo que respecta a la lectura en Internet. La búsqueda de información a través de la red o la comunicación a través de herramientas de correo electrónico, chats, etc. lleva a que los alumnos lean en Internet con una frecuencia elevada, registrándose un 73,79\% 
de lectores frecuentes -leen diaria o semanalmente-. También despierta algo más de interés la lectura de revistas, que es practicada por un $46,32 \%$ de los sujetos con una frecuencia igual o superior a una o dos veces por semana.

\subsection{Modalidades de lectura según los hábitos del alumnado}

Teniendo en cuenta la frecuencia con que leen los alumnos y alumnas, hemos llevado a cabo una reducción de los diferentes tipos de lectura para identificar modalidades genéricas. Con este propósito, hemos codificado los cuatro niveles de la escala de frecuencia con valores numéricos que van de 1 a 4 . Las puntuaciones asignadas a los sujetos participantes han sido sometidas a un análisis de componentes principales, seguido de una rotación varimax para clarificar la interpretación de los factores obtenidos. Previamente, se ha valorado la adecuación de la matriz de correlaciones para la aplicación del análisis factorial, obteniéndose una medida de adecuación de muestreo de Kaiser-Meyer-Olkin que alcanza el valor 0,67, el cual puede considerarse aceptable, y un estadístico chi cuadrado $=28.071,67$ en el test de esfericidad de Barlett, que permitiría rechazar con $\mathrm{p}<0,001$ la hipótesis nula de ausencia de correlaciones entre las variables.

La solución factorial resultante tras el análisis arroja dos factores que explican un $43,34 \%$ de la varianza total. La saturación de cada una de las variables en los dos factores, una vez realizada la rotación, se muestra en la tabla 3, en la que para simplificar la presentación se han suprimido los pesos factoriales inferiores a 0,30 .

\begin{tabular}{|l|c|c|}
\hline \multirow{2}{*}{\multicolumn{1}{|c|}{ Variables }} & \multicolumn{2}{c|}{ Componente } \\
\cline { 2 - 3 } & 1 & 2 \\
\hline Cuentos o novelas cortas &, 780 & \\
\hline Libros (juveniles, deportes, viajes...) &, 679 & \\
\hline Tebeos &, 594 & \\
\hline Internet & &, 649 \\
\hline Juegos de preguntas y respuestas & &, 615 \\
\hline Revistas & &, 578 \\
\hline Periódicos & &, 563 \\
\hline
\end{tabular}

Tabla 3. Matriz de componentes rotados. 
De acuerdo con los resultados obtenidos, se han identificado dos factores o componentes. El primero de ellos agrupa la lectura de cuentos o novelas cortas, libros (juveniles, deportes, viajes...) y tebeos, de tal manera que una puntuación alta en este factor implica una frecuencia elevada en la lectura de este tipo de materiales. Este factor se asocia por tanto a la lectura recreativa, que tiene como objeto la literatura preferentemente juvenil y que supone un modo de ocupar el tiempo libre de los escolares. El segundo de los factores es saturado por la lectura en Internet, en juegos de preguntas y respuestas, revistas y periódicos. Una puntuación factorial alta se corresponde con la lectura frecuente de este tipo de materiales, de modo que en este caso podríamos hablar de una lectura instrumental, al servicio de la comunicación, el acceso a la información o el desarrollo de actividades lúdicas que no tienen como finalidad intrínseca el disfrute con la lectura.

\subsection{Competencias básicas en función de hábitos de lectura}

El análisis de los niveles que logran los estudiantes en las competencias básicas según sus hábitos lectores se ha realizado a partir de las puntuaciones factoriales alcanzadas en lectura recreativa y lectura instrumental. Ambas modalidades de lectura han sido categorizadas en cuatro niveles de frecuencia, tomando como puntos de corte los cuartiles de sus respectivas distribuciones. Así, para cada modalidad de lectura, el total de estudiantes que han participado en el estudio queda dividido en cuatro grupos de tamaño similar, a los que hemos asignado respectivamente una frecuencia baja, media-baja, media-alta y alta en lectura recreativa o en lectura instrumental, según el caso.

En la tabla 4 se recogen las puntuaciones medias logradas en las competencias matemática, lingüística y científica por cada uno de los cuatro grupos considerados en función de su puntuación en el factor lectura recreativa, y esas mismas puntuaciones han sido representadas mediante diagrama de barras en la figura 1. Los resultados muestran una clara tendencia a que cuanto más elevada sea la puntuación en este factor mayor sea la media lograda en las tres competencias, tendencia que resulta algo más acusada en el caso de las competencias lingüística y científica. 


\begin{tabular}{|l|c|c|c|c|}
\hline \multirow{2}{*}{ Competencia } & \multicolumn{4}{|c|}{$\begin{array}{c}\text { Frecuencia de lectura recreativa } \\
\text { (cuentos o novelas, libros, tebeos) }\end{array}$} \\
\cline { 2 - 5 } & Baja & Media-baja & Media-alta & Alta \\
\hline Matemática & 491,06 & 497,55 & 510,23 & 521,06 \\
\hline Comunicación lingüística & 476,63 & 497,95 & 514,97 & 527,99 \\
\hline $\begin{array}{l}\text { Conocimiento e interacción } \\
\text { con mundo físico y natural }\end{array}$ & 479,01 & 495,48 & 511,62 & 527,14 \\
\hline
\end{tabular}

Tabla 4. Competencia media del alumnado en función del hábito de lectura recreativa (cuentos o novelas cortas, libros, tebeos)

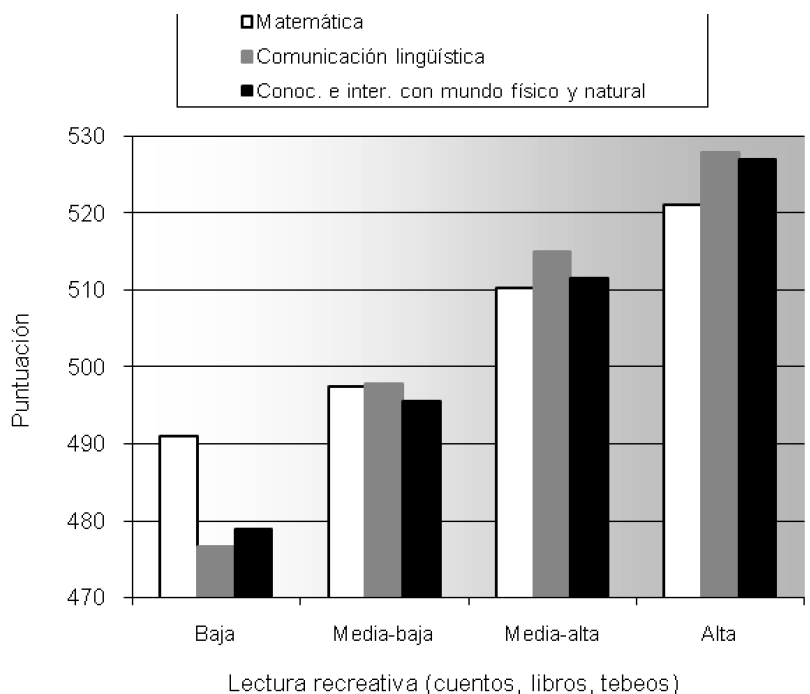

Figura 1. Competencias básicas del alumnado en función del hábito de lectura recreativa (cuentos o novelas, libros, tebeos)

Considerando los grupos que surgen a partir del factor lectura instrumental, hemos calculado las puntuaciones medias en las tres competencias medidas (ver tabla 5). A las puntuaciones bajas en este factor corresponden competencias medias claramente por debajo del 500, si bien no hay una tendencia clara ascendente para el resto de los niveles de puntuación en lectura instrumental. Esto queda reflejado en la similitud de las alturas alcanzadas 
por las barras mediante las que se han representado las puntuaciones medias (ver figura 2), todas ellas en el rango comprendido entre las puntuaciones 500 y 510 .

\begin{tabular}{|l|c|c|c|c|}
\hline \multirow{2}{*}{\multicolumn{1}{|c|}{ Competencia }} & \multicolumn{4}{|c|}{$\begin{array}{c}\text { Frecuencia de lectura recreativa } \\
\text { (cuentos o novelas, libros, tebeos) }\end{array}$} \\
\cline { 2 - 5 } & Baja & Media-baja & Media-alta & Alta \\
\hline Matemática & 498,26 & 507,98 & 506,47 & 507,44 \\
\hline Comunicación lingüística & 491,98 & 509,41 & 507,49 & 509,00 \\
\hline $\begin{array}{l}\text { Conocimiento e interacción } \\
\text { con mundo físico y natural }\end{array}$ & 499,00 & 508,68 & 504,64 & 501,41 \\
\hline
\end{tabular}

Tabla 5. Competencia media del alumnado en función del hábito de lectura instrumental (prensa, juegos, Internet)

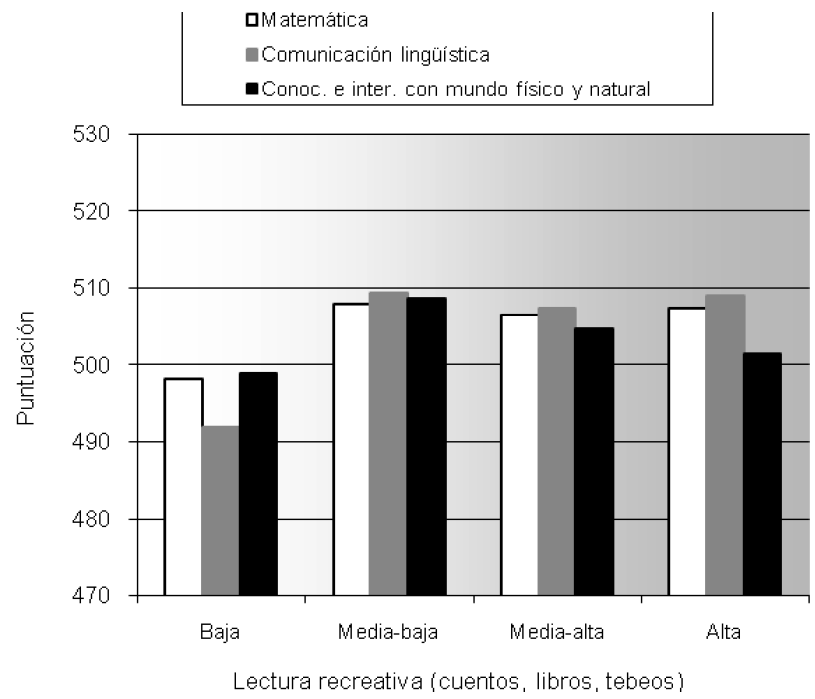

Figura 2. Competencias básicas del alumnado en función del hábito de lectura instrumental (prensa, juegos, Internet) 


\section{CONCLUSIONES Y REFLEXIONES FINALES}

Los hábitos lectores de los estudiantes se ven marcados por el avance imparable de las nuevas tecnologías en nuestra sociedad. En la actualidad los textos escritos se presentan en diferentes formatos, de tal modo que la lectura tradicional de libros, revistas, prensa o documentos diversos conviven con nuevas formas escritas, como son las que se basan en textos presentados a través de Internet, el correo electrónico, los chats y otras formas de transmisión de mensajes escritos. El lector joven se está habituando a prácticas de lectura nuevas y singulares, que pasan por la hipertextualidad y la lectura en pantalla (Domínguez y Sádaba, 2005). Entre los resultados destacados del presente estudio puede señalarse que la lectura en Internet es la forma de lectura más frecuente entre el alumnado que inicia tercero de Educación Secundaria Obligatoria. En cierto modo, estos resultados apuntan en la dirección de hallazgos de estudios recientes, en los que se ha concluido que el uso de Internet por parte de los estudiantes, tanto con fines académicos como recreativos, está empezando a ocupar el tiempo que en otra época éstos habrían dedicado a la lectura (Mokhtari, Reichard y Gardner, 2009).

En cuanto a la relación entre aprendizaje escolar y hábitos lectores, reiteradamente constatada en estudios previos, los resultados aquí obtenidos nos llevan a hacer alguna matización en función de las modalidades de lectura que consideremos. Los mayores niveles de desarrollo en las competencias básicas del alumnado se registran cuando los sujetos poseen el hábito de leer como forma de experiencia literaria, dirigida fundamentalmente al disfrute personal. En cambio, la realización frecuente de una lectura instrumental, orientada a la adquisición de información, a la comunicación o a la participación en diferentes contextos no se corresponde del mismo modo con altos niveles de aprendizaje.

Por tanto, si a través de la lectura se pretende favorecer en los estudiantes el desarrollo de la competencia lectora y, mediante ésta, la adquisición de aprendizajes escolares, parece conveniente recomendar el desarrollo de hábitos lectores centrados en materiales literarios. La razón para que se aprecie esta relación entre la lectura recreativa y el aprendizaje puede estar en los procesos de comprensión lectora que aquélla genera. Mientras que otros tipos de texto requieren del lector la captación de información explícita contenida en el mismo, los textos literarios exigen un mayor grado de inferencia, interpretación e integración de ideas. A menudo, los autores literarios construyen sus textos con la intención de sugerir, de llevar al lector a la interpretación y a inferir significados implícitos. Y además, los textos literarios se distancian claramente de otros tipos de texto en lo que respecta a la riqueza de vocabulario, corrección de la estructura gramatical, construcción 
del discurso y otros elementos textuales, susceptibles de ser apreciados y valorados por el lector, y que contribuyen al desarrollo de la competencia del sujeto para la lectura y, en consecuencia, para la adquisición de aprendizajes.

Asumiendo el desarrollo de los hábitos de lectura en los jóvenes como un objetivo a alcanzar, habría de contemplarse la posibilidad de intervención desde diferentes contextos, que incluyen el ámbito de las políticas en materia de educación y cultura, el ámbito de las instituciones escolares y el ámbito de las familias del alumnado.

Tratando de elevar las tasas de lectura registradas entre los ciudadanos de nuestro país, y como reacción ante los resultados adversos obtenidos en las sucesivas evaluaciones que se han realizado sobre la competencia lectora de nuestros estudiantes, las administraciones públicas a niveles nacional, autonómico o local vienen insistiendo en la puesta en marcha de programas de fomento de la lectura que se concretan, entre otras actuaciones, en la dotación de recursos para las bibliotecas públicas o en la realización de campañas de sensibilización y animación a la lectura. La propia Ley Orgánica de Educación ya recoge como objetivo el "fomento de la lectura" (art. 2) subrayando como meta de la Educación Primaria el desarrollo de hábitos lectores. De cara a conseguir esta meta, se señala la necesidad de dedicar un tiempo diario a la lectura en los centros educativos, además de contar con bibliotecas escolares que contribuyan al fomento de la lectura y que funcionen como espacio abierto a la comunidad educativa.

Desde el contexto escolar, se adoptan decisiones curriculares para poner el énfasis en el aprendizaje de la lectura y en el desarrollo de hábitos lectores. La disponibilidad de bibliotecas de centro y de aula, dotadas con suficientes fondos bibliográficos, no sólo constituye un recurso para el aprendizaje sino también una vía para favorecer el desarrollo de hábitos lectores en el alumnado. Sin embargo, la mera existencia de este recurso no es suficiente, si no va acompañada de un uso efectivo del mismo. Al analizar factores asociados al bajo rendimiento lector de los escolares españoles en las evaluaciones internacionales, respecto a otros países participantes, se ha señalado un menor uso de las bibliotecas escolares y de aula, y un menor porcentaje de profesorado que ha recibido formación específica en didáctica de la lectura (Martín, 2008). Tratando de paliar esta situación, se han arbitrado medidas que utilizan a los centros como escenario para la intervención. En el caso de la Comunidad Autónoma Andaluza, donde se enmarca el estudio aquí presentado, se está implementando durante el período 20062010 el Plan de Lectura y Biblioteca, con la finalidad de impulsar el desarrollo de prácticas lectoras y habilidades intelectuales en el alumnado andaluz de Educación Infantil, Primaria y Secundaria. De acuerdo con este Plan, se espera que en 2010 en torno a 2500 centros integren la Red de 
Centros con Proyectos Lectores, recibiendo recursos económicos y formación para la puesta en marcha de tales proyectos.

Por último, el contexto familiar puede ser decisivo para el desarrollo de hábitos lectores en los jóvenes. Las características familiares, el nivel cultural de los padres y madres, los valores y los usos del tiempo libre pueden configurar un entorno estimulante propicio para que los hijos desarrollen el gusto por la lectura. La actitud positiva hacia la lectura es algo que los niños y niñas pueden desarrollar en su hogar cuando en éste se valora y se fomenta la lectura, se dedica tiempo a leer, se leen cuentos a los más pequeños o se intercambian opiniones sobre libros leídos (Fiz y otros, 2000a; Lammed y Olmsted, 1977; Strommen y Mates, 2004). En este sentido, se ha señalado la importancia que las actitudes y comportamientos de los integrantes de la unidad familiar en relación a la lectura tienen para la formación de los hábitos lectores infantiles (Fernández, García y Prieto, 1999; Moreno, 2002), y su repercusión sobre el rendimiento académico (Gil, 2009).

De cara a favorecer el papel de las familias en la adquisición del hábito lector, las propias instituciones escolares podrían intervenir activamente a través de las relaciones familia-escuela. Algunas actuaciones que contribuirían a fortalecer el "clima lector" de los hogares podrían ser: la inclusión, en los programas formativos dirigidos a padres y madres, de contenidos relativos a la importancia de la lectura en la familia para desarrollar la lectura en los hijos; la apertura de las bibliotecas escolares a la comunidad educativa en general, brindando a los padres y madres el acceso a los fondos bibliográficos de los centros; o la participación de los padres y madres en las actividades de animación a la lectura que se promueven desde los centros, habilitando de este modo un espacio más para la lectura compartida y para la conversación sobre las lecturas realizadas.

En definitiva, el desarrollo de hábitos lectores entre nuestros jóvenes constituye un claro objetivo a cuyo logro se puede contribuir desde diferentes ámbitos de intervención. El hábito lector no sólo es una fuente de disfrute o un medio para conseguir información, sino que propicia una mejora de la competencia lectora y otras habilidades cognitivas que están en la base y son el fundamento para un aprendizaje continuo a lo largo de la vida, capaz de maximizar el potencial de desarrollo de los recursos humanos de un país e incrementar la calidad de vida de los ciudadanos. 


\section{NOTAS}

1. Este trabajo presenta resultados obtenidos en el marco de la Evaluación de Diagnóstico en Andalucía, realizada durante el curso 2008/2009, y es fruto del contrato de investigación firmado entre la Consejería de Educación y Ciencia y la Universidad de Sevilla. 


\section{REFERENCIAS BIBLIOGRÁFICAS}

Anderson, R.C., Wilson, P.T. y Fielding, L.G. (1988). Growth in reading and how children spend their time outside of school. Reading Research Quarterly, 23, 285-303.

Conlon, E.G.; Zimmer, M.J.; Creed, P.A. y Tucker, M. (2006). Family history, selfperceptions, attitudes and cognitive abilities are associated with early adolescent reading skills. Journal of Research in Reading, 29 (1), 11-32.

Cromley, J.G. (2009). Reading Achievement and Science Proficiency: International Comparisons from the Programme on International Student Assessment. Reading Psychology. 30 (2), 89-118.

Cunningham, A.E. y Stanovich, K.E. (1991). Tracking the unique effects of print exposure in children: Associations with vocabulary, general knowledge, and spelling. Journal of Educational Psychology, 83, 264-274.

Domínguez, M. y Sádaba, I. (2005). Transformaciones en las prácticas culturales de los jóvenes. De la lectura como ocio y consumo a la fragmentación neotecnológica. Revista de Estudios de Juventud, 70, 23-37.

European Comission (2007). European cultural values. Special Eurobarometer 278. Disponible en: http://ec.europa.eu/public_opinion/archives/ebs/eb s_278_en.pdf [consulta 2009, 17 de diciembre].

Fernández, N. (2005). La lectura entre las prácticas culturales de los jóvenes en España. Revista de Estudios de Juventud, 70, 13-21.

Fernández, V.; García, M. y Prieto, J. (1999). Los hábitos de lectura en Es- paña: características sociales, educativas y ambientales. Revista de Educación, 320, 379-390.

FGEE (2009). Barómetro de hábitos de lectura y compra de libros. Disponible en: http://www.federacioneditores.org /0_Resources/Documentos/NP_Lectura_TercerTrimestre_2009.pdf [consulta 2009, 17 de diciembre].

Fiz, M.R.; Goicoechea, M.J.; Ibiricu, O. y Olea, M.J. (2000a). Los hábitos de lectura y su relación con otras variables. Huarte de San Juan. Filología y Didáctica de la Lengua, 5, 7-31.

Fiz, M.R.; Goicoechea, M.J.; Ibiricu, O. y Olea, M.J. (2000b). La comprensión lectora y su repercusión en el rendimiento escolar. Huarte de San Juan. Filología y Didáctica de la Lengua, 6-7, 75-108.

Gil, J. (2009). Hábitos y actitudes de las familias hacia la lectura y competencias básicas del alumnado. Revista de Educación, 350, 301-322.

González, A. (1996). Comprensión lectora y rendimiento académico. Revista Galega de Psicopedagoxía, 9 (13), 209224.

Lammed, L. y Olmsted, P. (1977). Family reading habits and children's progress in reading. Annual Meeting of the International Reading Association. Miami Beach, Florida.

Leppanen, U.; Aunola, K. y Nurmi, J.E. (2005). Beginning readers' reading performance and reading habits. Journal of Research in Reading, 28 (4), 383-399.

Linnakylä, P. y Välijärvi, J. (2006). Rendimiento de los estudiantes finlandeses en PISA. Las claves del éxito en lectura. Revista de Educación, (extra), 227-235. 
Martín, E. (2008). El papel de la lectura en el sistema educativo, en Millán, J.A. (Coord.) La lectura en España. Informe 2008. Madrid: Fundación Germán Sánchez Ruipérez y Federación de Gremios de Editores de España, 123-136.

Meneghetti, C.; Carretti, B. y De Beni, R. (2006). Components of reading comprehension and scholastic achievement. Learning and Individual Differences, 16 (4), 291-301.

Mokhtari, K.; Reichard, C.A. y Gardner, A. (2009). The impact of Internet and television use on the reading habits and practices of college students. Journal of Adolescent and Adult Literacy, 52 (7), 609-619.

Molina, L. (2006). Lectura y educación: los hábitos lectores y su repercusión académica en la ESO. Ocnos. Revista de estudios sobre lectura, 2, 103-120.

Moreno, E. (2002). La familia como institución sociocultural: su papel en la adquisición de los hábitos lectores. Portularia. Revista de Trabajo Social, 2, 309-324.

Mullis, I.; Kennedy, A.M.; Martin, M.O. y Sainsbury, M. (2006). Estudio internacional de progreso en comprensión lectora. PIRLS 2006. Marcos teóricos y especificaciones de evaluación. Madrid: IEA-MEC.
Nolen, S. B. (2003). Learning environment, motivation, and achievement in high school science. Journal of Research in Science Teaching, 40 (4), 347-368.

O’Reilly, T., \& McNamara, D. S. (2007). The impact of science knowledge, reading skill, and reading strategy knowledge on more traditional "highstakes" measures of high school students' science achievement. American Educational. Research Journal, 44 (1), 161-196.

Savolainen, H.; Ahonen, T.; Aro, M.; Tolvanen, A. y Holopainen, L. (2008). Reading comprehension, word reading and spelling as predictors of school achievement and choice of secondary education. Learning and Instruction, 18 (2), 201-210.

Strommen, L.T. y Mates, B.F. (2004). Learning to love reading: interviews with older children and teens. Journal of Adolescent and Adult Literacy, 48 (3) 188-200.

Topping, K J. Samuels, J. Paul, T. (2007). Does practice make perfect? independent reading quantity, quality and student achievement. Learning and Instruction, 17 (3), 253-264. 


\section{PALABRAS CLAVE}

Hábitos lectores, rendimiento académico, educación secundaria.

\section{KEY WORDS}

Reading habits, academic performance, secondary education.

\section{PERFIL ACADÉMICO Y PROFESIONAL DEL AUTOR}

Javier Gil Flores, Doctor en Filosofía y Ciencias de la Educación por la Universidad de Sevilla, donde trabaja como Catedrático de Universidad, adscrito al área de Métodos de Investigación y Diagnóstico en Educación. Ha desarrollado diferentes estudios y ha publicado trabajos en el ámbito de la evaluación de competencias básicas del alumnado de Educación Primaria y Educación Secundaria Obligatoria.

Dirección del autor: C/ San Blas, 21 41003 - Sevilla. e-mail: jflores@us.es

Fecha de Recepción del Artículo: 04. Enero. 2010

Fecha de Aceptación del Artículo: 26. Marzo. 2010 